\title{
Gobernanza de la prevención social de la violencia y la delincuencia

\author{
El caso de Nuevo León
}

\author{
Governance of Socio-Economic Crime Prevention:The Case of Nuevo Leon
}

José Andrés Sumano Rodríguez*

Resumen: El presente texto analiza los procesos de gobernanza de los programas y acciones en materia de prevención social de la violencia y la delincuencia para el caso de Nuevo León. Se describen los principales actores involucrados en dichos procesos, los mecanismos deliberativos para la toma decisiones, la interacción entre los análisis racionales (policy analysis) y los ajustes mutuos de intereses (policy sciences) y el marco legal vigente en la materia. El texto destaca la complejidad de la gobernanza en materia de prevención social de la violencia y la delincuencia en el contexto mexicano. El texto también destaca la insuficiencia del gobierno para dar respuesta al problema de la violencia y la delincuencia en el contexto mexicano.

Palabras clave: gobernanza, prevención social, seguridad ciudadana, Nuevo León, políticas públicas.

Abstract: This text analyses the processes of governance pertaining the programs for socioeconomic crime and violence prevention for the case of Nuevo Leon. The text incorporates a discussion regarding the main actors involved in those processes, the deliberative mechanisms for decision-making, the interaction between policy analysis and policy sciences on this topic and the legal framework involved. The text highlights the complexity of governance regarding socio-economic crime prevention in the Mexican context. The text also highlights the insufficiency of government for responding to the challenges of crime and violence in the Mexican context.

Keywords: governance, socio-economic prevention, citizen security, Nuevo Leon, public policy.

*José Andrés Sumano Rodríguez es profesor investigador-Cátedra Conacyt en El Colegio de la Frontera Norte, Carretera Escénica Tijuana-Ensenada Km. 18.5, San Antonio del Mar, 22560, Tijuana, Baja California. Tel: (664) 631 6300. Correo-e: andressumano@colef.mx. oRcID: https://orcid.org/0000-0001-80308643.

Artículo recibido el 6 de junio de 2020 y aceptado para su publicación el 25 de junio 2021.

http://dx.doi.org/10.29265/gypp.v31i1.1016 


\section{SOBRE EL CASO}

- ste caso tiene como propósito ser una herramienta para la docencia dentro del ¿paradigma pedagógico de aprendizaje a través de discusión (Barnes et al., 1994). En este sentido, se busca profundizar en los conceptos de gobernanza, coproducción y administración pública deliberativa a través del estudio del caso de la prevención social de la violencia en Nuevo León. El objetivo es vincular el conocimiento con la acción, de manera que las alumnas y alumnos puedan ir descubriendo los conceptos mediante su aplicación en casos de la vida real. El caso se encuadra, dentro de la tipología elaborada por Lynn (1999) y retomada por Arellano y Vega (2019), en los casos de tipo concept-application que tienen como propósito "que los estudiantes mejoren su apreciación de un concepto teórico, analítico o de proceso". Conforme a las recomendaciones de Arellano y Vega (2019), se sugiere su uso posterior a la revisión de algunas lecturas teóricas en la materia. En este sentido, al final del caso se proporcionan dos bibliografías, la primera con los materiales consultados para la elaboración del caso y la segunda con lecturas recomendadas para la introducción teórica a la materia de estudio. La metodología utilizada para la elaboración del caso consistió en observación participante durante un periodo de dieciocho meses dentro de la Subsecretaría de Prevención y Participación Ciudadana de Nuevo León. Aunado a lo anterior, es pertinente señalar que el caso está escrito en forma de vivencia. Para facilitar la discusión y el aprendizaje activo a través del caso, se incluye una serie de preguntas divididas conforme a la tipología sugerida por Barnes et al. (1994) en: a) preguntas de análisis, b) preguntas de acción y c) preguntas de reflexión.

\section{INTRODUCCIÓN}

En febrero de 2016, María Elena Domínguez fue invitada por Jaime Rodríguez Calderón, gobernador de Nuevo León, a colaborar como subsecretaria de Prevención y Participación Ciudadana en su gobierno. En octubre de 2015 había tomado posesión el nuevo gobernador de Nuevo León, Jaime Rodríguez Calderón, tras una elección histórica, en la cual por primera ocasión un candidato sin partido ganaba una elección para gobernador en México (Alanís, 2015). El margen de victoria para Jaime Rodríguez Calderón en la elección y el contexto de ser el primer gobernador sin partido generaban altas expectativas entre los ciudadanos de Nuevo León respecto a los posibles resultados de su gobierno. ${ }^{1}$ El antecesor de María

\footnotetext{
${ }^{1}$ Jaime Rodríguez obtuvo 48.86 por ciento de los votos, mientras que el candidato del pan, Felipe de Jesús Cantú, obtuvo 22.52 por ciento de los votos y la candidata del PRI, Ivonne Álvarez, obtuvo 23.56 por ciento de los votos.
} 
Elena Domínguez en la Subsecretaría de Prevención y Participación Ciudadana había renunciado al cargo por motivos personales tras haber durado sólo cuatro meses en el cargo (Milenio Digital, 2016).

\section{APUNTES TEÓRICOS EN MATERIA DE PREVENCIÓN SOCIAL DE LA VIOLENCIA Y LA DELINCUENCIA}

La corriente de pensamiento que ha dominado el debate sobre prevención social de la violencia y la delincuencia en México durante los últimos años ha sido el modelo epidemiológico, también conocido como paradigma desarrollista y de curso de vida (DOF, 2014b; Wong y Manning, 2017). La aplicación de dicho modelo en el país tuvo su momento cumbre con la implementación del Programa Nacional para la Prevención Social de la Violencia y la Delincuencia (Pronapred) entre 2014 y 2018. El auge del modelo epidemiológico en las políticas públicas de prevención de la violencia y la delincuencia derivó de un esfuerzo de la administración del expresidente Enrique Peña Nieto por diferenciarse del enfoque reactivo y punitivo que caracterizó a la administración que le antecedió. El nuevo enfoque buscaba transitar de un modelo reactivo a uno preventivo que atendiera las causas de raíz de la violencia y la delincuencia (DOF, 2014b).

El modelo epidemiológico es una adaptación derivada de las ciencias de la salud que pretende entender a la violencia y la delincuencia como enfermedades. Dicho enfoque considera que la violencia y delincuencia se comportan de forma similar a una epidemia, por lo que el crecimiento o disminución de los niveles de violencia y delincuencia están condicionados a una serie de factores de riesgo y factores de protección (DOF, 2014b; Wong y Manning, 2017). En la medida en que se fortalezcan los factores de protección será mas difícil el desarrollo de violencia y delincuencia. A su vez, en la medida en que se disminuyan los factores de riesgo también se aminoran las posibilidades de desarrollar violencia y delincuencia. Los factores de riesgo y protección están clasificados en distintos niveles que van desde lo individual hasta lo comunitario (Wong y Manning, 2017). En este sentido, algunos factores de riesgo pueden ser las adicciones, el embarazo temprano, la deserción escolar, los entornos deteriorados, el desempleo, la falta de espacios públicos y la poca cohesión comunitaria. A su vez, algunos factores de protección pueden ser: el desarrollo de habilidades para la vida, el sentido de pertenencia, la crianza positiva, los espacios públicos de calidad, el desarrollo de capacidades para el trabajo y un adecuado alumbrado público.

Desde la visión epidemiológica, las políticas públicas deben estar dirigidas a disminuir los factores de riesgo y aumentar los factores de protección, atendiendo 
de esta forma las causas de raíz de la violencia y la delincuencia y no simplemente reaccionando a ellas a través de mecanismos como la policía (DOF, 2014b; Wong y Manning, 2017). Algunos ejemplos de políticas públicas de este tipo son los talleres de habilidades para la vida para adolescentes en conflicto con la ley, las campañas para la prevención de adicciones, el otorgamiento de becas para disminuir la deserción escolar, la recuperación de espacios públicos, los talleres de capacitación para el trabajo, la instalación de alumbrado público y la terapia cognitivo-conductual para prevenir la violencia familiar.

Sin embargo, la principal crítica al modelo epidemiológico radica en cuestionar la eficacia de dichas acciones en entornos de alta violencia. En este tipo de contextos, autores como Thomas Abt (2019) han señalado que las políticas de prevención social de la violencia no tendrán la eficacia deseada si no se atiende la violencia misma primero, pues consideran difícil que un joven que teme por su vida pueda aprovechar adecuadamente los talleres de capacitación para el trabajo o las campańas para la prevención de adicciones. Otra crítica radica en la debilidad de las teorías del cambio asociadas a varios programas de prevención social de la violencia y la delincuencia. En el caso mexicano, se llegaron a implementar en el país programas como huertos urbanos o clases de zumba que no establecían una clara teoría del cambio respecto a cómo se contribuía a reducir la violencia y la delincuencia (Chapa y Ley, 2015).

\section{APUNTES TEÓRICOS SOBRE GOBERNANZA, COPRODUCCIÓN Y ADMINISTRACIÓN PÚBLICA DELIBERATIVA}

En el enfoque weberiano, la solución a los problemas públicos partía de burocracias profesionales (Weber, 2008). Desde el Estado, a través de la eficacia indiferente representada en la burocracia, se podía dar respuesta a las problemáticas sociales. Dicha concepción estaba cimentada en la confianza en la capacidad técnico-racional de los seres humanos para dar respuesta a problemas complicados. Sin embargo, la realidad actual es compleja, no complicada, y la racionalidad humana es limitada (Ostrom, 2015; Simon, 1997). En este sentido, los problemas públicos terminan siendo construcciones sociales, por lo que es inevitable que las respuestas también lo sean (Wildavsky, 1987).

En ese entorno de problemas complejos y racionalidad limitada, el Estado y su eficacia indiferente no bastan. La cuestión deja de ser la eficacia y eficiencia del Estado, para pasar a ser la suficiencia del Estado (Aguilar, 2006). Desde esa perspectiva, Vincent Ostrom (2007) planteó la idea de gobernanza policéntrica que sugiere soluciones distintas a la burocracia para los problemas públicos, así 
como actores distintos al Estado en la solución de los mismos. Por su parte, Elinor Ostrom (2015) propondría que el diálogo y la confianza entre actores locales, con el Estado o sin él, puede llevar a las comunidades a la solución de problemas públicos complejos. Esto implica profundizar más allá de la eficacia y las soluciones gerenciales propuestas por la nueva gestión pública.

El impacto de la idea de complejidad en el abordaje de los problemas públicos no fue menor, implicó asumir los límites de la racionalidad y la planeación técnica, la posibilidad de consecuencias no intencionadas de la acción, que el Estado no tiene todas las respuestas ni es el único actor y que la solución a los problemas públicos complejos requiere de coproducción con otros actores sociales (Scott, 1999; Simon, 1997). El cambio de paradigma resaltó la importancia de las redes de políticas públicas, de los liderazgos relacionales sobre los liderazgos gerenciales y del diálogo como mecanismo para balancear objetivos poco claros y a veces contrapuestos (Brugué et al., 2015; Sumano, 2020; Tarragó y Brugué-Torruella, 2015).

En el paradigma de la gobernanza toma una mayor relevancia la capacidad de coordinación respecto a la profundidad del análisis técnico (Aguilar, 2006). La confianza entre actores resalta como un factor clave para lograr la colaboración (Sumano, 2020; Tarragó y Brugué-Torruella, 2015). Ante la insuficiencia del Estado, la deliberación y la cooperación permiten un mejor abordaje de problemas complejos, reconociendo que muchas veces las soluciones no necesariamente serán óptimas, pero sí llevarán a un escenario mejor que el previo. Desde esta perspectiva, se reconoce y se hace parte de la solución que tanto la definición de los problemas, como las soluciones, contienen una carga valorativa significativa en lo que Deborah Stone (2011) denominó policy paradox. Por lo tanto, las políticas públicas derivadas de la deliberación y el diálogo en un entorno de confianza tendrán mayores probabilidades de éxito (Brugué et al., 2015; Sumano, 2020; Tarragó y Brugué-Torruella, 2015).

Lo anterior no significa que el Estado y el análisis técnico racional no mantengan un rol fundamental en la solución de problemas públicos. El Estado sigue siendo un actor relevante, pero en un entramado amplio de actores que asumen distintos roles en diferentes contextos (Klijn, 1998). Por su parte, la evidencia es un insumo fundamental en la construcción de argumentos para la persuasión y en la mejora constante de las políticas públicas (Majone, 2005).

\section{CONTEXTO DE VIOLENCIA EN NUEVO LEÓN}

En 2010 y 2011, la ciudad de Monterrey experimentó los momentos mas díficiles de la crisis de seguridad que azotó en esos años varias partes del país, y que 
tuvo su máxima expresión en la zona con el incendio en el Casino Royal (Vega, 2011). Un número significativo de habitantes de la ciudad dejaron la misma ante la ola de violencia que azotaba la ciudad por la estrategia de seguridad federal enfocada en una confrontación directa con los grupos criminales, las disputas por la plaza entre grupos delictivos y el incremento en las prácticas de extracción de rentas (Durin, 2012). En respuesta a la emergencia, el sector privado, sociedad civil y gobierno generaron diversos proyectos conjuntos para enfrentar la crisis, siendo el más emblemático la creación de Fuerza Civil (Mendoza y Montero, 2015). Desde entonces, la incidencia delictiva en la zona metropolitana de Monterrey ha ido disminuyendo. Hoy, las policías de municipios como San Pedro Garza García, Guadalupe o General Escobedo son consideradas modelos de éxito y varios municipios de la zona metropolitana se encuentran entre los municipios con mayor percepción de seguridad (Excélsior, 2019). Fue en ese contexto, junto a las expectativas creadas por el triunfo del primer gobierno independiente de Nuevo León, que María Elema Domínguez asumía el cargo.

\section{SUBSECRETARÍA DE PREVENCIÓN Y PARTICIPACIÓN CIUDADANA}

La Subsecretaría de Prevención y Participación Ciudadana que ahora dirigiría María Elena Domínguez había sido creada en el gobierno de Rodrigo Medina a efecto de dar respuesta a las dinámicas institucionales del gobierno federal, en donde el presidente Enrique Peña Nieto había creado la Subsecretaría de Prevención y Participación Ciudadana al interior de la Secretaría de Gobernación y le había encomendado implementar el Programa Nacional de Prevención del Delito. En el caso de Nuevo León, la Subsecretaría fue creada al interior de la Secretaría de Seguridad Pública y, a diferencia del modelo federal, concentraba también el Centro Estatal de Prevención del Delito (Periódico Oficial, 2013).

La Subsecretaría estaba dividida en tres direcciones: La Dirección del Centro Estatal de Prevención del Delito, la Dirección del Modelo Integral de Intervención Comunitaria y la Dirección de Atención Ciudadana (Periódico Oficial, 2013). En la Subsecretaría colaboraban un aproximado de 50 empleados que se dividían en esas tres direcciones. Los principales programas que operaba la Subsecretaría cuando María Elena Domínguez tomó posesión eran el Centro de Atención Integral para Adolescentes en Riesgo (Caipa), el Centro de Formación de Relaciones Humanas (Ceforeh), el Programa Padres y Madres Promotoras de la Paz, el Programa Dare (Drugs Abuse Resistence Education, en inglés) y el Programa de formación de Comités Ciudadanos de Prevención. 
El presupuesto estatal para la Subsecretaría de Prevención y Participación Ciudadana constaba de 38 millones de pesos, los cuales se repartían entre sueldos y papelería $(D O F, 2015 a)$. En este sentido, la Subsecretaría no contaba con recursos estatales para la implementación de nuevos programas y acciones. Incluso, en muchas ocasiones los mismos empleados de la Subsecretaría aportaban de sus propios recursos para trasladarse o comprar la papelería necesaria para poder implementar sus programas. La implementación de nuevas acciones o programas dependía en gran medida de recursos ajenos al gobierno del estado, en particular de los subsidios federales.

\section{PRONAPRED Y OTROS SUBSIDIOS O FONDOS FEDERALES}

En el año 2014, en seguimiento a su propuesta de campaña de atender los factores estructurales de la violencia y la delincuencia en México y dentro del eje México en Paz del Plan Nacional de Desarrollo 2013-2018, el presidente Enrique Peña Nieto creó el Programa Nacional para la Prevención Social de la Violencia y la Delincuencia. En dicho programa se contemplaba un subsidio a los estados para la implementación de acciones en prevención social de la violencia y la delincuencia en el marco de cinco objetivos: 1) incrementar la corresponsabilidad de la ciudadanía y actores sociales en la prevención social mediante su participación y desarrollo de competencias, 2) reducir la vulnerabilidad ante la violencia y la delincuencia de las poblaciones de atención prioritaria, 3) generar entornos que favorezcan la convivencia y la seguridad ciudadanas, 4) fortalecer las capacidades institucionales para la seguridad ciudadana en los gobiernos municipales/ delegacionales, estatales y federal, y 5) asegurar la coordinación entre las dependencias y entidades de la administración pública federal para la implementación de programas de prevención social (DOF, 2014b). Asimismo, se estableció una serie de factores de riesgo a los que deberían estar dirigidas las acciones de prevención social implementadas con dicho subsidio (DOF, 2014b):

- Individuales: adicciones, intolerancia, baja capacidad de resolución de conflictos, embarazo temprano y violencia en el noviazgo.

- Familiares: baja cohesión familiar, estilos parentales coercitivos y agresivos, bajo nivel de ingreso familiar y participación de familiares en actividades ilegales.

- Grupales: participación en grupos de pares involucrados en actividades con violencia, drogas o delitos.

- Educativos: deserción escolar y violencia escolar. 
- Comunitarios: deterioro del capital social, entornos deteriorados, fácil acceso a armas y cultura de la ilegalidad.

- Sociales y culturales: carencias esenciales para el desarrollo humano integral, falta de opciones laborales y falta de opciones de estudio.

Para el ańo 2016, el primero de la gestión de María Elena Domínguez al frente de la Subsecretaría de Prevención y Participación Ciudadana, los recursos provenientes del subsidio Pronapred para el estado de Nuevo León habían disminuido significativamente, pasando de 136 millones de pesos en el año 2014 y 129 millones de pesos en el año 2015 a 69 millones de pesos en 2016 (DOF, 2014a, 2015b, 2016). Lo anterior era parte de un recorte generalizado al subsidio derivado de la crisis fiscal que enfrentaba el gobierno federal.

En lo que respecta al Fondo de Aportaciones para la Seguridad Pública (FASP), el cual contemplaba como uno sus programas con prioridad nacional el desarrollo de capacidades en las instituciones locales para el diseño de políticas públicas destinadas a la prevención social de la violencia y la delincuencia con participación ciudadana en temas de seguridad pública, el entonces secretario general de Gobierno y coordinador del Gabinete de Seguridad, Lic. Manuel González Flores, decidió que los cerca de 35 millones de pesos destinados para dicho programa en el año 2016 deberían ser destinados a la construcción de un centro de acceso a la justicia para las mujeres, dejando sin presupuesto del faSP a la Subsecretaría de Prevención y Participación Ciudadana para la implementación de programas de prevención social (SESNSP, 2016).

En lo concerniente al Fortaseg, un subsidio dirigido a los municipios y que contemplaba también recursos para el programa de prioridad nacional de desarrollo de capacidades en las instituciones locales para el diseño de políticas públicas destinadas a la prevención social de la violencia y la delincuencia con participación ciudadana en temas de seguridad pública, pero al cual el gobierno del estado tenía derecho a 50 por ciento de los recursos derivado de su corresponsabilidad en el patrullaje del municipio de Monterrey, el entonces secretario general de Gobierno y coordinador del Gabinete de Seguridad, Lic. Manuel González Flores, decidió destinar cero pesos a dicho programa en el año 2016, dejando sin presupuesto de Fortaseg a la Subsecretaría de Prevención y Participación Ciudadana para la implementación de nuevos programas (SESNSP, 2016). Es importante mencionar que el FASP y el Fortaseg eran administrados por el Secretariado Ejecutivo del Sistema Nacional de Seguridad Pública, que contaba con el Centro Nacional de Prevención del Delito y Participación Ciu- 
dadana, y el Pronapred era administrado por la Subsecretaría de Prevención y Participación Ciudadana de la Secretaría de Gobernación.

\section{MARCO LEGAL Y MECANISMOS DELIBERATIVOS}

Derivado de la asignación de recursos para prevención social de la violencia y la delincuencia para el año 2016, María Elena Domínguez sabía que debía maximizar la eficacia y eficiencia de los recursos correspondientes al subsidio Pronapred 2016 en un contexto de alta incertidumbre y bajo la premisa de lo que lo realizado por el gobierno es insuficiente, por si sólo, para reducir los niveles de inseguridad y violencia. En este sentido, Los lineamientos para el otorgamiento de apoyos a las entidades federativas en el marco del Programa Nacional de Prevención del Delito para el año 2016 establecían la necesidad de instaurar una Comisión Interinstitucional para la Prevención Social de la Violencia y la Delincuencia en la que participaran los representantes de los municipios de la zona metropolitana de Monterrey con polígonos de atención prioritaria en el marco del Pronapred, miembros de organizaciones de la sociedad civil y representantes de instituciones académicas. Dicha comisión debía aprobar la propuesta de distribución de recursos

CUADRO 1. Polígonos de Pronapred 2016

Polígonos que atendió Pronapred en 2016

- Polígono Metroplex en Apodaca (colonia Metroplex)

- Polígono Bellavista en Cadereyta (colonias Bella Vista, Jardines de Cadereyta, Real de Cadereyta, Fomerrey Cadereyta y Los Encinos)

- Polígono Villas de San Francisco en Escobedo (colonias Villas de San Francisco y Villas de San Miguel)

- Polígono Urbivillas del Prado en García (colonia Urbivilla del Prado)

- Polígono Tierra Propia en Guadalupe (colonia Tierra Propia)

- Polígono Los Naranjos de Juárez (colonias Ampliación Rancho Viejo, Arboleda de los Naranjos, Los Valles 1 y 2 sector, y Villa de los Naranjos)

- Polígono Norte de Monterrey (Tierra Propia F. 35, Valle de San Martín)

- Polígono Sur de Monterrey (Cerro de la Campana, Burócratas Municipales)

- Polígono La Fama III en Santa Catarina (colonias Lomas de la Fama y Fama III)

- Polígono El Barrial en Santiago (comunidad El Barrial)

- Polígono Paseo de Nogalar en San Nicolás (colonias Lago de Chapultepec, Paseo de Nogalar, Floridos Bosques del Nogalar)

- Polígono San Pedro 400 en San Pedro (colonia San Pedro 400)

Fuente: Información proporcionada por la Subsecretaría de Prevención y Participación Ciudadana de Nuevo León. 
y las acciones a implementar para poder proceder a la aprobación de la propuesta por parte de la Subsecretaría de Prevención y Participación Ciudadana de la Secretaría de Gobernación (DOF, 2016).

En el caso de Nuevo León, el Pronapred para 2016 debía contemplar acciones en 12 polígonos de atención prioritaria distribuidos en 11 municipios de la zona metropolitana de Monterrey (DOF, 2016).

En este sentido, la Comisión Interinstitucional para la Prevención Social de la Violencia y la Delincuencia en Nuevo León decidió la siguiente distribución de recursos (gráfica 1). Es preciso notar que en las reuniones de la Comisión Interinstitucional para la Prevención Social de la Violencia y la Delincuencia se requería la asistencia de 50 por ciento +1 a efecto de poder contar con quórum legal. Asimismo, las decisiones eran aprobadas por mayoría simple. La Comisión Interinstitucional debía sesionar por lo menos dos veces al año, una al inicio del año para aprobar la propuesta de acciones y distribución de los recursos y otra a final

GRÁFICA 1. Distribución de recursos del Pronapred 2016

Monto total de los recursos Pronapred 2016 para la zona metropolitana de Monterrey: $\$ 69046257.15$ (la primera ministración corresponde a 80\% de los recursos)

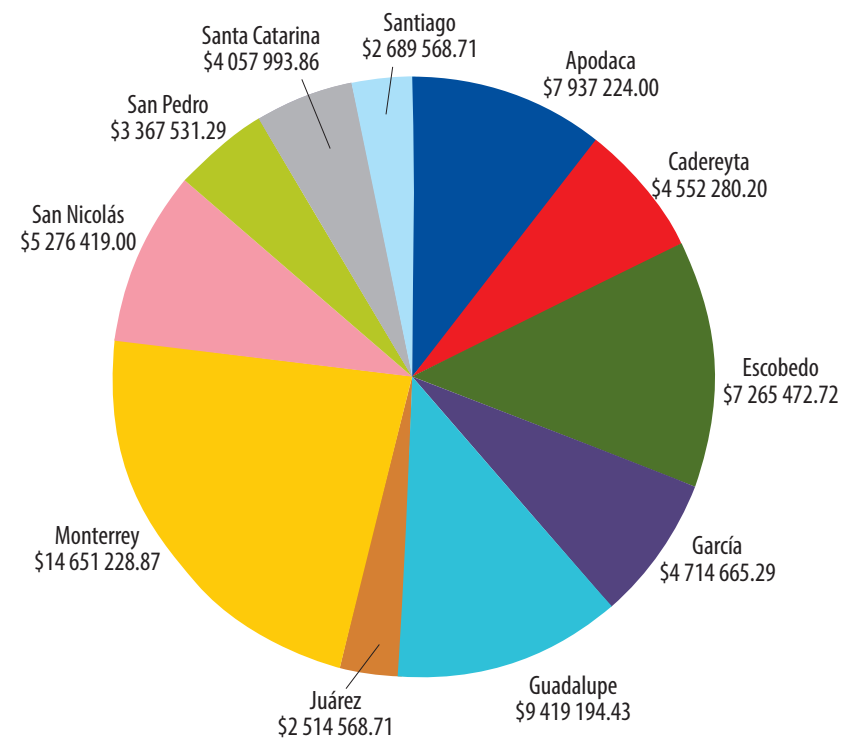

Fuente: Información proporcionada por la Subsecretaría de Prevención y Participación Ciudadana de Nuevo León. 
del año para la presentación, por parte de la Subsecretaría de Prevención y Participación Ciudadana, de los resultados de las acciones y, en su defecto, la firma del acta de cierre que era requerida conforme a los lineamientos para el otorgamiento de apoyos a las entidades federativas en el marco del Programa Nacional de Prevención del Delito para el ańo 2016 a efecto de dar por cerrado el ejercicio fiscal del programa en comento.

Aunado a lo anterior, el cierre del ejercicio fiscal 2016 requería el envío a la Subsecretaría de Prevención y Participación Ciudadana de la Secretaría de Gobernación de actas de entrega para cada una de las acciones contempladas en el Pronapred 2016 en las cuales firmaran tanto el gobierno municipal como el gobierno estatal la recepción de la acción a completa satisfacción. En este sentido, era necesario que ambos niveles de gobierno estuvieran de acuerdo con las acciones y aprobaran su entrega a efecto de dar por concluida la acción $(D O F, 2016)$.

\section{OTROS ACTORES}

Las acciones de prevención social de la violencia y la delincuencia con recursos del subsidio del Pronapred son sometidas a procesos de licitación para poder definir el proveedor responsable de la implementación (DOF, 2000). En este sentido, las organizaciones de la sociedad civil que tradicionalmente habían trabajado temas cercanos a la prevención social de la violencia y la delincuencia en Nuevo León y en el resto del país incrementaron su activismo y presencia en los diversos foros relacionados con el Pronapred con dos objetivos: 1) ganar procesos de licitación correspondientes a Pronapred y 2) impulsar el financiamiento de las actividades que ya llevaban a cabo con recursos del Pronapred.

El caso de Nuevo León y del mandato de María Elena Domínguez en la Subsecretaría de Prevención y Participación Ciudadana de Nuevo León no fue la excepción. En este sentido, se estableció una mesa de diálogo con organizaciones de la sociedad civil neoleonesas como Vetsa A.C., Renace A.C., Supera A.C., Cifac A.C., entre otras, con la finalidad de establecer puentes de diálogo respecto a la transparencia y eficacia en los procesos de licitación y en la selección de las acciones de prevención social a implementar.

Otra organización con un rol preponderante en el contexto de la prevención social de la violencia y la delincuencia para el caso de Nuevo León era USAID (siglas en inglés de la Agencia de Estados Unidos para el Desarrollo Internacional) y su programa Juntos por la Prevención de la Violencia operado por la empresa Chemonics. El programa Juntos por la Prevención de la Violencia, que inició en el año 2016, a la par de la llegada de María Elena Domínguez a la Subsecretaría 
de Prevención y Participación Ciudadana de Nuevo León, tenía como propósito brindar apoyo técnico a nivel municipal con el objetivo de facilitar la implementación de acciones de prevención social de la violencia y la delincuencia basadas en evidencia. En el caso de Nuevo León, dicho programa solo operaba en tres municipios: Monterrey, Escobedo y Guadalupe. En este sentido, Juntos por la Prevención de la Violencia trabajaba activamente con dichos municipios para la instalación de gabinetes de prevención social, para el fortalecimiento de programas de prevención secundaria y terciaria dirigidos a jóvenes y en la capacitación de personal para implementar políticas de prevención social de la violencia y la delincuencia basadas en evidencia (USAID, 2018). Sin embargo, el enfoque estrictamente municipalista del programa no contemplaba la colaboración con la Subsecretaría de Prevención y Participación Ciudadana del estado, dificultando la cooperación entre niveles de gobierno para esos objetivos.

Por su parte, el Instituto Republicano Internacional iniciaba un nuevo programa enfocado a la prevención social de la violencia y la delincuencia a la par que María Elena Domínguez tomaba posesión como subsecretaria de Prevención y Participación Ciudadana. El Instituto Republicano Internacional buscaba implementar un programa que generara una herramienta digital que facilitara la transparencia y el combate a la corrupción en materia de prevención social (Lage et al., 2017). El interés de dicho instituto derivaba de los constantes reclamos de organizaciones de la sociedad civil por corrupción en la administración de los fondos del Pronapred. Sin embargo, la implementación de dichas herramientas y una mayor transparencia en la administración del presupuesto encontraba reticencias en algunos municipios.

La Fundación Carlos Slim generó también en ese momento un programa digital de capacitación denominado Diplomado en Prevención Social de las Violencias. A través de dicho programa buscaban capacitar a todo el personal de los gobiernos estatales y municipales que desempeñaba tareas de prevención social. Para lo anterior, generaron un acuerdo con la Subsecretaría de Prevención y Participación Ciudadana de la Secretaría de Gobernación y con el Centro Nacional de Prevención del Delito y Participación Ciudadana a efecto de que las entidades y municipios estuvieran obligados a destinar recursos del Programa de Fortalecimiento para la Seguridad (Fortaseg) correspondientes al año 2017 para la implementación de dicho programa de capacitación (Brito, 2017). El programa respondía a la necesidad de capacitar a un número creciente de funcionarios dedicados a la prevención social tras la creación del Pronapred.

Finalmente, el Tecnológico de Monterrey, a través de su programa Distrito Tec, estaba impulsando acciones de prevención social de la violencia y la delincuencia 
en colonias cercanas al campus de la institución en Monterrey. En este sentido, el Tecnológico de Monterrey estaba coordinando a diferentes actores para diseñar un plan integral de intervención en la zona de La Campana-Altamira, entre los cuales buscaba incorporar a la Subsecretaría de Prevención y Participación Ciudadana de Nuevo León (Distrito Tec, 2018). En los últimos años, la institución

CUADRO 2. Mapeo de actores

\begin{tabular}{|c|c|c|c|c|}
\hline Actor & Principal interés & Recursos & Acciones & Rol \\
\hline $\begin{array}{l}\text { Organizaciones } \\
\text { de la sociedad } \\
\text { civil en Nuevo } \\
\text { León }\end{array}$ & $\begin{array}{l}\text { Obtener } \\
\text { financiamiento para } \\
\text { desarrollar proyectos } \\
\text { de prevención } \\
\text { social desde sus } \\
\text { organizaciones. }\end{array}$ & $\begin{array}{l}\text { Experiencia en labores de } \\
\text { prevención social en las } \\
\text { comunidades. Legitimidad } \\
\text { en las comunidades. }\end{array}$ & $\begin{array}{l}\text { Cabildeo de sus modelos } \\
\text { y programas ante } \\
\text { instancias financiadoras. } \\
\text { Implementación a nivel } \\
\text { de calle de programas de } \\
\text { prevención social. }\end{array}$ & Neutral \\
\hline $\begin{array}{l}\text { USAID- } \\
\text { Chemonics }\end{array}$ & $\begin{array}{l}\text { Desarrollar } \\
\text { capacidades al } \\
\text { interior de los } \\
\text { gobiernos locales } \\
\text { para implementar } \\
\text { programas de } \\
\text { prevención social } \\
\text { basados en evidencia. }\end{array}$ & $\begin{array}{l}\text { Presupuesto disponible } \\
\text { para el acompañamiento } \\
\text { y asesoramiento a } \\
\text { municipios. } \\
\text { Conocimientos } \\
\text { especializados en el } \\
\text { acompañamiento a } \\
\text { programas de prevención } \\
\text { social de la violencia. }\end{array}$ & $\begin{array}{l}\text { Elaborar manuales y } \\
\text { guías. Brindar talleres y } \\
\text { asesoría. Desarrollo de } \\
\text { herramientas para la } \\
\text { prevención social. }\end{array}$ & Neutral \\
\hline $\begin{array}{l}\text { Instituto } \\
\text { Republicano } \\
\text { Internacional }\end{array}$ & $\begin{array}{l}\text { Desarrollar una } \\
\text { herramienta } \\
\text { para mejorar la } \\
\text { transparencia en } \\
\text { la administración } \\
\text { del presupuesto de } \\
\text { prevención social. }\end{array}$ & $\begin{array}{l}\text { Presupuesto disponible } \\
\text { para el desarrollo e } \\
\text { implementación de la } \\
\text { herramienta. }\end{array}$ & $\begin{array}{l}\text { Proveer la herramienta } \\
\text { a estados y municipios. } \\
\text { Asesoría sobre la } \\
\text { herramienta. }\end{array}$ & Aliado \\
\hline $\begin{array}{l}\text { Fundación } \\
\text { Carlos Slim }\end{array}$ & $\begin{array}{l}\text { Mejorar las } \\
\text { capacidades de } \\
\text { los gobiernos } \\
\text { para la prevención } \\
\text { social a través de la } \\
\text { capacitación. }\end{array}$ & $\begin{array}{l}\text { Plataforma tecnológica. } \\
\text { Presupuesto disponible } \\
\text { para impartir talleres y } \\
\text { cursos. }\end{array}$ & $\begin{array}{l}\text { Impartir diplomados } \\
\text { en prevención social de } \\
\text { la violencia a personal } \\
\text { dedicado a dichas tareas } \\
\text { en gobiernos estatales y } \\
\text { municipales. }\end{array}$ & Aliado \\
\hline $\begin{array}{l}\text { Tecnológico de } \\
\text { Monterrey }\end{array}$ & $\begin{array}{l}\text { Mejorar la seguridad } \\
\text { en los alrededores del } \\
\text { campus Monterrey. }\end{array}$ & $\begin{array}{l}\text { Instalaciones educativas } \\
\text { disponibles. Legitimidad } \\
\text { en las comunidades } \\
\text { vecinas al campus. } \\
\text { Capacidades técnicas } \\
\text { para el asesoramiento y } \\
\text { acompañamiento. }\end{array}$ & $\begin{array}{l}\text { Brindar talleres y cursos. } \\
\text { Facilitar instalaciones. }\end{array}$ & Aliado \\
\hline
\end{tabular}

Fuente: Elaboración propia. 
educativa había mostrado un creciente interés en la prevención social de la violencia, en particular a través de la prevención situacional de la violencia en los alrededores del campus, tras el asesinato de dos de sus estudiantes a manos del ejército.

\section{NUEVO LEÓN EN PAZ}

En ese contexto, María Elena Domínguez debía tratar de responder a los retos de violencia y delincuencia de Nuevo León desde la Subsecretaría de Prevención y Participación Ciudadana. Ella sabía que ante lo limitado de los recursos y la complejidad de los retos en la materia, aun si lograba maximizar la eficacia y eficiencia de la Subsecretaría, no sería suficiente para dar respuesta a la problemática de la violencia y la delincuencia en Nuevo León. En este sentido, requería coproducir la prevención social de la violencia y la delincuencia junto con los actores estatales involucrados e interesados en la materia. En los términos de Luis F. Aguilar (2006), la implementación de herramientas de la nueva gestión pública que tenían como objetivo maximizar la eficiencia del gobierno no iban a ser suficientes para la demanda en la materia. Se requería partir del paradigma de la gobernanza a efecto de poder dar respuesta al problema de la insuficiencia del gobierno en la materia (Aguilar, 2006).

De esta manera, María Elena Domínguez buscó generar un esquema de respuesta a dicha problemática desde la hipótesis sostenida por autores como Habermas (1981), Hajer y Wagennar (2003), y Font (2003), respecto a que la solución de problemas complejos como la violencia y la delincuencia requiere de diálogo e intercambio de recursos entre las diferentes partes de la administración pública y entre la administración y su entorno. En este sentido, estructuró una estrategia denominada Nuevo León en Paz con base en las siguientes dos premisas:

1. Los gobiernos que construyen soluciones a problemas complejos como la violencia y la delincuencia a través de la colaboración, la comunicación y el diálogo tienen mayores posibilidades de éxito (Agranoff y McGuire, 2003; Innes y Booher, 2003).

2. La generación de confianza entre las diferentes áreas del gobierno y entre este y la ciudadanía debe ser un objetivo de la gestión a efecto de poder alcanzar gobiernos más eficaces (Child y Faulkner, 1998; Lane y Bachmann, 1998).

La estrategia Nuevo León en Paz tenía como eje fundamental la coproducción de la prevención social de la violencia y la delincuencia y en este sentido se construyeron las siguientes alianzas y programas: 
- Se generó una alianza con el Tecnológico de Monterrey y la empresa Cemex para poder llevar a cabo un Macro Mural de $25000 \mathrm{~m}^{2}$ en la colonia La Campana y una Ciudad Mural en la colonia Altamira. En este sentido, la empresa Cemex donaría la pintura requerida para ambos proyectos, el Tecnológico de Monterrey brindaría asesoría técnica y hospedaje al personal del colectivo encargado del proyecto y la Subsecretaría de Prevención y Participación Ciudadana de Nuevo León pagaría, a través del Pronapred, los honorarios del colectivo contratado. Ambos proyectos se enmarcaban dentro de un esquema de prevención situacional, pero también incorporaban aspectos de fortalecimiento del capital social y cohesión comunitaria. El referente era el macro mural realizado previamente en Pachuca, Hidalgo, por el colectivo Germen Crew.

- En otro ámbito de prevención, se generó una alianza con la Universidad Autónoma de Nuevo León, en concreto con su Facultad de Música, y con todos los municipios beneficiarios de Pronapred en el ańo 2016 en la zona metropolitana de Monterrey para implementar un proyecto común de prevención primaria a través de la música. En este sentido, la Subsecretaría de Prevención y Participación Ciudadana de Nuevo León y los municipios beneficiarios del

FIGURA 1. Macro Mural de Palmitas en Pachuca, Hidalgo

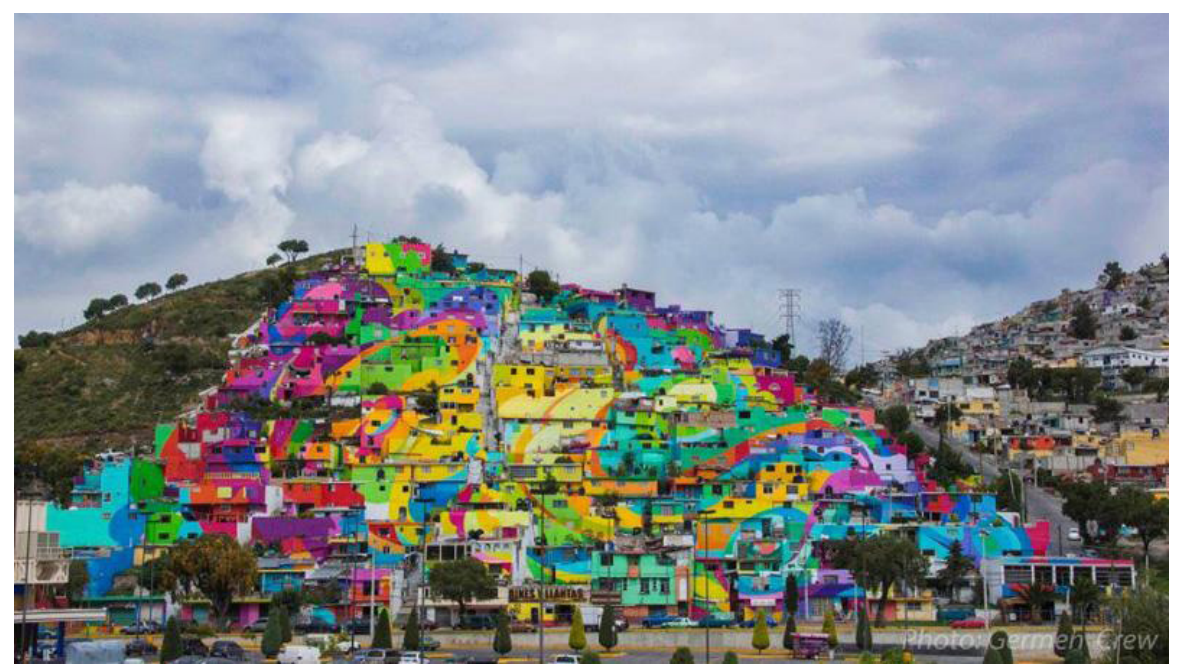

Fuente: Germen Crew. 
Pronapred en la zona metropolitana de Monterrey pagarían, a través del Pronapred, la compra de instrumentos musicales, uniformes y equipamiento y la Facultad de Música de la Universidad Autónoma de Nuevo León aportaría a los maestros y sus instalaciones. El objetivo de la intervención era conformar una red de orquestas de prevención con 25000 jóvenes y nińos de los polígonos del Pronapred a efecto de alejarlos de la violencia y la delincuencia a través de la práctica de la música.

- Una tercera alianza que se generó tenía que ver con la prevención terciaria. En este sentido, la Subsecretaría de Prevención y Participación Ciudadana de Nuevo León se asoció con la empresa Femsa para generar un programa de acompañamiento en libertad. El programa buscaba brindar capacitación en habilidades para la vida y oportunidades de empleo para las personas liberadas del sistema penitenciario. La Subsecretaría de Prevención y Participación Ciudadana brindaba terapias psicológicas y talleres de habilidades para la vida a las personas liberadas del sistema penitenciario y Femsa se comprometía a brindarles un empleo en sus tiendas oxxo a través de un proceso de contratación rápido.

- Finalmente, la cuarta alianza tenía como objetivo desarrollar un programa de prevención secundaria dirigido a jóvenes pertenecientes a pandillas en la zona

FIgURA 2. Foto de la Orquesta de Prevención de El Barrial

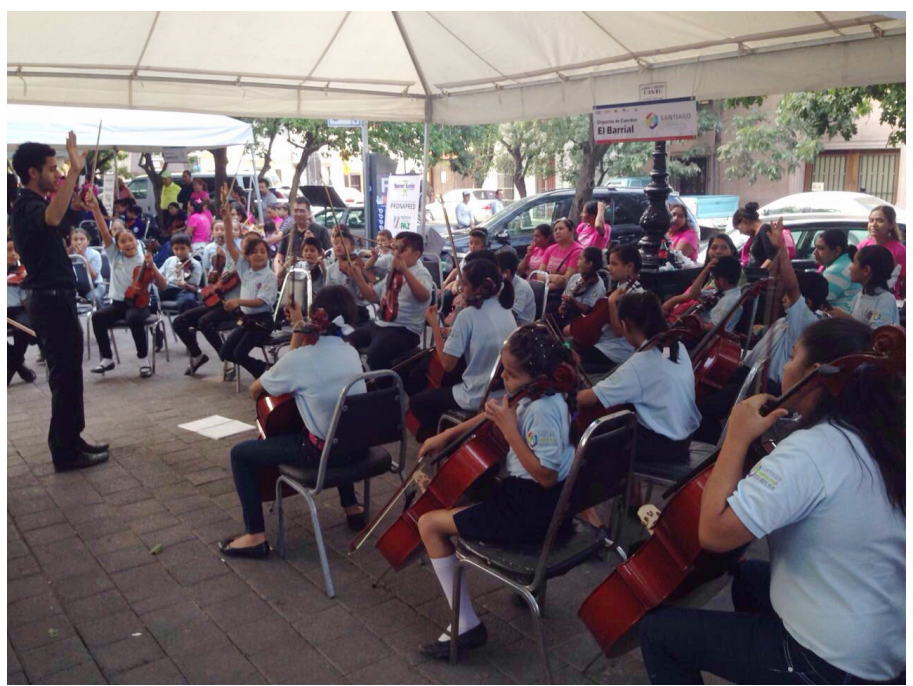

Fuente: Facultad de Música de la UANL. 
norte de Monterrey. En este sentido, la Subsecretaría de Prevención y Participación Ciudadana se asoció con la Fundación Incluyendo México y con la empresa Katcon para financiar el proyecto Nacidos para Triunfar. Cada una de las partes aportaría una tercera parte de los recursos económicos necesarios para implementar el proyecto, el cual consistía en generar un proceso de acercamiento con 100 pandillas de la zona norte de Monterrey, posteriormente firmar treguas de paz entre ellas, luego implementar una escuela de líderes dirigida a esos jóvenes y, finalmente, brindarles oportunidades de empleo.

Es importante mencionar que, aunado a estas cuatro alianzas, la Subsecretaría de Prevención y Participación Ciudadana de Nuevo León implementaba otros proyectos y programas. Sin embargo, estas cuatro alianzas formaban los ejes de la estrategia Nuevo León en Paz.

\section{COMPLICACIONES}

El ejercicio de los recursos provenientes del subsidio Pronapred implicaba la licitación de los proyectos a contratar (fuera en la modalidad compra directa, invitación a cuando menos tres personas o licitación pública nacional) conforme a la Ley de Adquisiciones, Arrendamientos y Servicios del Sector Público (DOF, 2000). Dicho proceso de licitación no dependía de la Subsecretaría de Prevención y Participación Ciudadana de Nuevo León, sino de la recién creada Secretaría de Administración del Gobierno de Nuevo León que tenía como propósito concentrar todos los procesos de licitación de las diferentes dependencias gubernamentales. En este sentido, la Subsecretaría de Prevención y Participación Ciudadana debía solicitar el proceso de licitación correspondiente a la Dirección General de Administración de la Secretaría de Seguridad Pública, que a su vez era la responsable de solicitar y monitorear el procedimiento con la Secretaría de Administración. Dicho procedimiento solo podía iniciarse una vez que los fondos correspondientes al Pronapred 2016 fueran depositados a la Tesorería del Estado por parte del Gobierno Federal. El tiempo estimado de duración del proceso desde la solicitud hasta la declaratoria de un ganador del proceso de licitación era aproximadamente de tres meses. En este sentido, si los recursos eran depositados en abril o mayo (como había ocurrido en años anteriores), las organizaciones ganadoras del proceso de licitación podrían estar empezando a implementar en agosto. La única opción legal disponible para evitar dichos procesos de licitación era implementar los proyectos a través de convenios de colaboración con instituciones públicas autónomas o descentralizadas, como universidades o institutos. 
Aunado a lo anterior, en septiembre de 2016, la Secretaría de Gobernación informó que haría un recorte a los recursos prometidos para el Pronapred 2016 de un 13 por ciento. Dicho recorte se aplicaría a la segunda ministración (Holguín, 2016). En este sentido, María Elena Domínguez tenía que replantear su programa de trabajo tomando en consideración que contaba con 13 por ciento menos recursos económicos. Lo anterior era de particular relevancia, pues un buen número de acciones se habían contemplado tomando en consideración el monto original, lo que implicaba recortar metas o de plano descartar acciones o programas. Es importante mencionar que el recorte de metas o la eliminación de acciones o programas debía ser consensuada con los municipios beneficiarios del Pronapred para el año 2016.

En ese contexto de ajuste presupuestal y tiempos apretados, María Elena Domínguez también se enfrentaba con una constante confusión a nivel directivo al interior del gobierno del estado respecto a las diferencias entre prevención social de las violencias y la delincuencia y desarrollo social. La entonces secretaria de Desarrollo Social del Estado, Luz Natalia Berrun, había solicitado al secretario de Seguridad Pública del Estado, Gral. Cuauhtémoc Antúnez, el traspaso de la Subsecretaría de Prevención y Participación Ciudadana a la Secretaría de Desarrollo Social del Estado. El secretario general de Gobierno, Manuel González, había solicitado a María Elena Domínguez alinear las estrategias y acciones de prevención social de la violencia y la delincuencia con el programa de trabajo de la Secretaría de Desarrollo Social. Sin embargo, los objetivos de la Secretaría de Desarrollo Social enfocados en reducción de la pobreza no eran en muchas ocasiones compatibles con los objetivos de reducción de factores de riesgo o de índices delictivos en ciertas zonas que estaban planteados para los subsidios federales en materia de prevención. Incluso, las zonas de atención prioritaria a las que enfocaban sus acciones las áreas de desarrollo social no eran necesariamente las mismas que las zonas de atención prioritaria en materia de prevención social de la violencia y la delincuencia.

\section{SITUACIONES CONFLICTIVAS}

Aunado al conflicto conceptual entre la Secretaría de Seguridad Pública y la Secretaría de Desarrollo Social sobre la pertinencia de subordinar las acciones de prevención social de la violencia a la agenda para la disminución de la pobreza, el cual impactaba negativamente en la colaboración interinstitucional y tenía consecuencias respecto a los objetivos a cumplir por la política de prevención social de la violencia, se sumaban los conflictos políticos y presupuestales. Conforme se 
iba acercando la temporada electoral para renovar presidencias municipales en Nuevo León, aumentaban las tensiones entre los diferentes niveles de gobierno, a la par que las limitantes presupuestales obligaban a elegir entre diversos objetivos y programas.

El panorama electoral poco a poco iba tomando mayor relevancia. En particular se generaron tensiones con el Ayuntamiento de Monterrey, pues dado el carácter federal de los recursos Pronapred, pero al ser administrados por el gobierno estatal y estar destinados al ámbito municipal, cada instancia de gobierno buscaba que las acciones realizadas se le atribuyeran a los ojos de los electores. Aunado a la competencia por la autoría de las acciones realizadas, también se acrecentaba la tensión entre los criterios de efectividad en materia de prevención social de la violencia y rentabilidad política de las acciones a implementar.

En otro frente, los recortes presupuestales y los atrasos en las licitaciones generaban inconformidad entre las organizaciones de la sociedad civil por la disminución de los tiempos para implementar los programas. Además, las limitaciones presupuestales generaban un debate intenso entre niveles de gobierno y socios implementadores del sector privado y la sociedad civil respecto a la definición de prioridades. Los diferentes actores involucrados en la política de prevención social se encontraban ante la necesidad de balancear objetivos en muchas ocasiones contrapuestos, con los desacuerdos que dicho proceso implica. Sin embargo, las exigencias ciudadanas por mejores resultados en materia de prevención de la violencia y delincuencia no disminuían.

\section{BRECHAS DE IMPLEMENTACIÓN}

María Elena Domínguez decidió, en conjunto con los representantes de los municipios beneficiarios del Pronapred 2016, que la mejor decisión frente al recorte presupuestal era eliminar algunas acciones en lugar de recortas metas. Lo anterior bajo el argumento de que era preferible implementar menos acciones, pero con los alcances planteados originalmente, que fragmentar aún más el escaso recurso económico.

Sin embargo, los procesos de licitación correspondientes al Pronapred 2016 para Nuevo León se retrasaron y la mayoría de las organizaciones ganadoras empezaron a implementar las acciones en el mes de octubre. Lo anterior se debió a retrasos en el depósito de los fondos por parte de la Secretaría de Haciendo y Crédito Público, a disputas dentro del gobierno estatal sobre los procesos de licitación y al retraso imperante en el área de adquisiciones de la Secretaría de Administración del Gobierno de Nuevo León respecto a los procesos de licitación en general. 
Debido a lo anterior, la mayoría de las organizaciones ganadoras de los procesos de licitación del Pronapred tuvieron solo tres meses para implementar las acciones, afectando negativamente la calidad de las mismas. Sin embargo, sí hubo acciones que lograron una mayor permanencia e impacto. Las acciones que se implementaron bajo convenio con la Universidad Autónoma de Nuevo León, por la estructura misma de los convenios, permitieron que las mismas comenzaran antes que el resto de las acciones y que tuvieran una duración de un ańo. Lo anterior impactó positivamente en los resultados de las mismas. Dichas acciones fueron la Red de Orquestas Juveniles de Prevención organizada con la Facultad de Música de la Universidad Autónoma de Nuevo León (UANL) que alcanzó la meta de 25000 niños y jóvenes en 26 orquestas en 12 polígonos de alta incidencia delictiva y el Centro de Atención Integral para Adolescentes (CAIPA) organizado con la Facultad de Psicología de la UANL y que logró atender a más de 800 jóvenes en riesgo o en conflicto con la ley a través las oficinas instaladas en cada uno de los municipios beneficiarios del Pronapred 2016.

En muchos casos, María Elena Domínguez tuvo que llegar a acuerdos con las diferentes organizaciones que estaban implementado acciones en el marco del Pronapred 2016 para que las acciones continuaran y lograran sus objetivos mas allá de la fecha oficial de conclusión de la acción establecida conforme a los lineamientos para el 31 de diciembre de 2016. En este sentido, un buen número de las acciones correspondientes a Pronapred 2016 tuvieron dos entregas, la entrega oficial requerida para el 31 de diciembre de 2016 y la entrega final que se realizó una vez que la acción cumplió con los objetivos planteados en el tiempo originalmente establecido.

\section{DESENLACE}

En mayo de 2017, el general Cuauhtémoc Antúnez, secretario de Seguridad Pública de Nuevo León renunció a su cargo (Maldonado, 2017). La renuncia del entonces secretario coincidió con la renuncia de su subordinada, María Elena Domínguez, a la Subsecretaría de Prevención y Participación Ciudadana del Estado. Además, la renuncia de ambos coincidió con la planeación del presupuesto en materia de prevención social de la violencia y la delincuencia para el ańo 2017. Es importante mencionar que la Secretaría de Hacienda y Crédito Público, en la propuesta de presupuesto de egresos de la federación para el año 2017, que posteriormente fue aprobada por la Cámara de Diputados, no incluyó recursos económicos para el subsidio Pronapred (Salazar, 2017). En este sentido, las entidades 
federativas y municipios perdieron su principal fuente de recursos para implementar proyectos de prevención de la violencia y la delincuencia. La decisión del gobierno federal de no incluir recursos para el subsidio Pronapred coincidió con una serie de recortes a diferentes programas federales derivado de una crisis fiscal a nivel federal.

En lo que respecta al subsidio Programa de Fortalecimiento para la Seguridad (Fortaseg), para el ańo 2017, el Secretariado Ejecutivo del Sistema Nacional de Seguridad Pública estableció una lista de 50 municipios prioritarios en materia de homicidios dolosos en el que incluyó a Monterrey. En este sentido, tanto el municipio de Monterrey como el gobierno del estado de Nuevo León debieron destinar los recursos correspondientes al Programa de Prioridad Nacional de Desarrollo de Capacidades en las instituciones locales para el diseńo de políticas públicas destinadas a la prevención social de la violencia y la delincuencia con participación ciudadana en temas de seguridad pública a la implementación de una estrategia para la reducción del homicidio doloso. Lo anterior daba un enfoque claro e integralidad a las acciones de prevención, pero dejaba fuera muchos aspectos de la prevención social que no estaban vinculados al homicidio doloso.

En lo que respecta al Fasp para el año 2017, María Elena Domínguez dejó aprobados los siguientes proyectos por un monto total de 5 millones de pesos: Programa de Acompańamiento en Libertad, Segunda Etapa del Macro Mural en el Cerro de la Campana, Segunda Etapa de Ciudad Mural en la Colonia Altamira y Campaña en Redes Sociales sobre Prevención Social. Los recursos para estos proyectos estaban muy limitados, por lo que el próximo subsecretario de Prevención y Participación Ciudadana debía generar las redes y apoyos necesarios a efecto de coproducir dichos proyectos y lograr una implementación exitosa (SESNSP, 2017).

Sin embargo, a un año de la salida de María Elena Domínguez de la Subsecretaría de Prevención y Participación Ciudadana de Nuevo León, dicha dependencia enfrenta una de las principales crisis de su corta historia. Ante el recorte en los presupuestos del Pronapred, la Red de Orquestas Juveniles de Prevención pasó a depender presupuestalmente del Instituto Estatal de las Mujeres, permitiendo su permanencia pero mermando su enfoque de prevención social. El Centro de Atención Integral para Adolescentes en Riesgo y/o Conflicto con la Ley pasó a depender presupuestalmente de la Secretaría de Desarrollo Social abonando a la confusión conceptual a nivel directivo entre desarrollo social y prevención social de las violencia. El Centro de Formación de Relaciones Humanas cerró sus actividades en el resto de los municipios y se mantiene operando con una sola oficina en las instalaciones de la Subsecretaría donde atienden todos los casos en materia 
de violencia contra las mujeres. El Macro Mural en el Cerro de la Campana tiene un progreso de 50 por ciento y ha pasado a depender de la Secretaría de Desarrollo Social del Estado donde le cambiaron de nombre a Colosal. La sucesora de María Elena Domínguez en el cargo, la Lic. Mónica Garza, apenas duró unos meses en el cargo y renunció para buscar una candidatura al Senado. En marzo de 2018 se anunció el nombramiento de un nuevo subsecretario de Prevención y Participación Ciudadana para el Estado quien deberá hacer frente a esta situación. Aunque el Presupuesto de Egresos de la Federación para el año 2018 sí contempló recursos para el subsidio Pronapred, estos fueron mucho menores a los contemplados en años anteriores, por lo que la política de prevención social de la violencia y la delincuencia para Nuevo León deberá redefinirse a partir de la premisa de que los recursos federales en la materia son sumamente escasos $(D O F, 2018)$. Gㅡㅡ

\section{REFERENCIAS}

Abt, T. (2019), Bleeding Out: The Devastating Consequences of Urban Violence and a Bold New Plan for Peace in the Streets, Nueva York, Basic Books.

Agranoff, R. y M. McGuire (2003), Collaborative Public Management, Washington, D.C., Georgetown University Press.

Aguilar, L.F. (2006), Gobernanza y gestión pública, Ciudad de México, Fondo de Cultura Económica.

Alanís, R. (2015), “'El Bronco' toma protesta como gobernador de NL", Milenio, 3 de octubre, disponible en: https://www.milenio.com/politica/el-bronco-toma-protesta-comogobernador-de-nl [fecha de consulta: 10 de agosto de 2020].

Arellano, D. y A. Vega (2019), Ilustraciones de la acción pública en el México contemporáneo: Estudios de caso para la docencia, Ciudad de México, Universidad Autónoma Metropolitana.

Barnes, L., C. Christensen, A. Hansen y T. Hansen (1994), "Teaching with Cases at the Harvard Business School”, en L. Barnes, C. Christensen y A. Hansen (eds.), Teaching and the Case Method: Text, Cases, and Readings, Nueva York, Harvard Business Press.

Brito, O. (2017), "Segob y fundación Carlos Slim firman convenio de colaboración", Milenio, 24 de abril, disponible en: https://www.milenio.com/politica/segob-fundacioncarlos-slim-firman-convenio-colaboracion [fecha de consulta: 10 de agosto de 2020].

Brugué, Q., R. Canal y P. Paya (2015), “¿Inteligencia administrativa para abordar 'problemas malditos'?: El caso de las comisiones interdepartamentales”, Gestión y Política Pública, XXIV(1), pp. 85-130.

Chapa, L. y S. Ley (2015), Prevención del delito en México: ¿Cuáles son las prioridades? Ciudad de México, México Evalúa. 
Child, J. y D. Faulkner (1998), Strategies of Cooperation: Managing Alliances, Networks, and Joint Ventures, Oxford, Oxford University Press.

DistritoTec (2018), "DistritoTec es el lugar donde se viven las grandes ideas", Monterrey, Tecnológico de Monterrey, disponible en: http://distritotec.itesm.mx/acerca/ [fecha de consulta: 12 de agosto de 2020].

DOF (Diario Oficial de la Federación) (2000), Ley de Adquisiciones, Arrendamientos y Servicios del Sector Público, disponible en: http://www.diputados.gob.mx/LeyesBiblio/ pdf/14_200521.pdf [fecha de consulta: 23 de junio de 2020].

DOF (Diario Oficial de la Federación) (2014a), Lineamientos para el otorgamiento de apoyos a las entidades federativas en el marco del Programa Nacional de Prevención del Delito, disponible en: https://www.dof.gob.mx/nota_detalle.php?codigo $=5332783 \& \mathrm{fec}$ $\mathrm{ha}=14 / 02 / 2014$ [fecha de consulta: 23 de junio de 2020].

DOF (Diario Oficial de la Federación) (2014b), Programa Nacional para la Prevención Social de la Violencia y la Delincuencia 2014-2018, disponible en: http://www.dof.gob.mx/ nota_detalle.php?codigo $=5343087 \&$ fecha $=30 / 04 / 2014$ [fecha de consulta: 22 de junio de 2020].

DOF (Diario Oficial de la Federación) (2015a), Ley de Egresos del Estado para el año 2016, disponible en: http://www.diputados.gob.mx/LeyesBiblio/abro/pef_2016/PEF_2016_ abro.pdf [fecha de consulta: 22 de marzo de 2020].

DOF (Diario Oficial de la Federación) (2015b), Lineamientos para el otorgamiento de apoyos a las entidades federativas en el marco del Programa Nacional de Prevención del Delito.

DOF (Diario Oficial de la Federación) (2016), Lineamientos para el otorgamiento de apoyos a las entidades federativas en el marco del Programa Nacional de Prevención del Delito.

DOF (Diario Oficial de la Federación) (2018), Lineamientos para el otorgamiento de apoyos a las entidades federativas en el marco del Programa Nacional de Prevención del Delito (2018), disponible en: https://dof.gob.mx/nota_detalle.php?codigo=5511236\&fec ha $=24 / 01 / 2018$ [fecha de consulta: 25 de mayo de 2020].

Durin, S. (2012), "Los que la guerra desplazó: Familias del noreste de México en el exilio", Desacatos, 38, pp. 29-42.

Excélsior (2019), "Innovador modelo de seguridad en Escobedo se aplicará a todo el país", Excélsior, 29 de junio, disponible: https://www.excelsior.com.mx/nacional/innovadormodelo-de-seguridad-en-escobedo-se-aplicara-a-todo-el-pais/1321572 [fecha de consulta: 10 de agosto de 2020].

Font, J. (2003), Public Participation and Local Governance, Barcelona, ICPS. Habermas, J. (1981), The Theory of Communicative Action, Boston, Beacon Press. 
Hajer, M. y H. Wagennar (2003), Deliverative Policy Analysis: Understanding Governance in the Network Society, Cambridge University Press.

Holguín, M.E. (2016), "Evitarán impacto de recortes de Pronapred”, El Siglo, 28 de julio, disponible en: https://www.elsiglodetorreon.com.mx/noticia/1247550.evitaran-impacto-de-recortes-de-pronapred.html [fecha de consulta: 9 de agosto de 2020].

Innes, J. y D. Booher (2003), “Collaborative Policymaking: Governance Through Dialogue”, en M.A. Hajer y H. Wagenaar (eds.), Deliverative Policy Analysis: Understanding Governance in the Network Society, Cambridge, Cambridge University Press.

Klijn, E.H. (1998), "Policy Networks: An Overview”, en W.J.M. Kickert, E.H. Klijn y J.F.M. Koppenjan (eds.), Managing Complex Networks, Londres, Sage.

Lage, F., N. Ruiz y M. García (2017), “Gobierno abierto y participación ciudadana: Antídotos para la corrupción”, Blog Democracy and Speaks, disponible en: https://www.democracyspeaks.org/blog gobierno-abierto-y-participaci\%C3\%B3n-ciudadana-ant $\%$ C3\%ADdotos-para-la-corrupci\%C3\%B3n [fecha de consulta: 7 de septiembre de 2020].

Lane, C. y R. Bachmann (1998), Trust Within and Between Organization, Oxford, Oxford University Press.

Lynn, L. (1999), Teaching and Learning with Cases: A Guide Book, Nueva York, Seven Bridges Press.

Majone, G. (2005), Evidencia, argumentación y persuasión en la formulación de politicas, Ciudad de México, Fondo de Cultura Económica.

Maldonado, O. (2017), “Anuncia gobernador salida del general Antúnez”, Milenio, 10 de mayo, disponible en: https://www.milenio.com/politica/anuncia-gobernador-salida-delgeneral-antunez [fecha de consulta: 12 de mayo de 2020].

Mendoza, H.E. y J.C. Montero (2015), “Gobernanza para la gobernabilidad: La construcción de 'Fuerza Civil', la nueva policía de Nuevo León”, Revista Mexicana de Análisis Político y Administración Pública, IV(1), pp. 103-128.

Milenio Digital (2016), "Renuncia funcionario de Seguridad Pública de NL", Milenio, 6 de febrero, disponible en: https:/www.milenio.com/estados/renuncia-funcionario-de-seguridad-publica-de-nl [fecha de consulta: 12 de mayo de 2020].

Ostrom, E. (2015), Governing the Commons: The Evolution of Institutions for Collective Action, Cambridge, Cambridge University Press.

Ostrom, V. (2007), The Intellectual Crisis in American Public Admnistration, Tuscaloosa, University Alabama Press.

Periódico Oficial (2013), Reglamento Interior de la Secretaría de Seguridad Pública, disponible en: http://sgi.nl.gob.mx/Transparencia_2015/Archivos/AC_0001_0004_01666920000001.pdf [fecha de consulta: 25 de mayo de 2020].

Salazar, C. (2017), “Diputados dejan en cero prevención del delito”, Reporte Índigo, 19 de 
enero, disponible en: https://www.reporteindigo.com/reporte/prevencion-delitos-pronapred-monterrey-escuela/ [fecha de consulta: 9 de julio de 2020].

Scott, J. (1999), Seeing Like the State: How Certain Schemes to Improve the Human Condition Have Failed, Nueva York, Yale University Press.

SESNSP (Secretariado Ejecutivo del Sistema Nacional de Seguridad Pública) (2016), Anexo del Convenio del Fondo de Aportaciones para la Seguridad Pública (FASP), 2016, Nuevo León, disponible en: https://www.gob.mx/sesnsp/documentos/anexos-de-subsidios2016?idiom=es [fecha de consulta: 25 de mayo de 2020].

SESNSP (Secretariado Ejecutivo del Sistema Nacional de Seguridad Pública) (2017), Anexo técnico del Fondo de Aportaciones para la Seguridad Pública, 2017, Nuevo León, diponible en: https://www.gob.mx/sesnsp/documentos/anexos-de-los-convenios-del-fondo-deaportaciones-para-la-seguridad-publica-fasp-2017?state=published [fecha de consulta: 25 de mayo de 2020].

Simon, H. (1997), Administrative Behavior: A Study of Decision Making Processes in Administrative Organizations, Nueva York, Free Press.

Stone, D. (2011), Policy Paradox: The Art of Political Decision Making, tercera edición, Nueva York, W.W. Norton \& Company.

Sumano, J.A. (2020), "Prevención del delito en la frontera norte a través de la administración pública deliberativa”, Gestión y Política Pública, XXIX(1), pp. 67-96.

Tarragó, D. y Q. Brugué-Torruella (2015), "La administración deliberativa: De la eficacia y la eficiencia a la inteligencia, y de la burocracia a la innovación”, Textos para Discusión de la CEPAL, 58.

USAID (Agencia de los Estados Unidos para el Desarrollo Internacional) (2018), Juntos por la prevención de la violencia: Reporte Anual, 2017-2018, disponible en: https://pdf.usaid. gov/pdf_docs/PA00TNMK.pdf [fecha de consulta: 7 de agosto de 2020].

Vega, A. (2011), "Terror en el Casino Royale de Monterrey; los Zetas, detrás del atentado", Excélsior, 26 de agosto, disponible en: https://www.excelsior.com.mx/2011/08/26/nacional/763911 [fecha de consulta: 14 de abril de 2020].

Weber, M. (2008), Economía y sociedad, Ciudad de México, Fondo de Cultura Económica. Wildavsky, A. (1987), Speaking Truth to Power: The Art and Craft of Policy Analysis, Nueva York, Routledge.

Wong, G. y M. Manning (2017), “Developmental Interventions”, en T. Prenzler (ed.), Understanding Crime Prevention: The Case Study Approach, Sidney, Australian Academic Press Group.

\section{LECTURAS RECOMENDADAS}

Aguilar, L.F. (2006), Gobernanza y gestión pública, Ciudad de México, Fondo de Cultura Económica. 
Brugué, Q., R. Canal y P. Paya (2015), “¿Inteligencia administrativa para abordar 'problemas malditos'?: El caso de las comisiones interdepartamentales", Gestión y Política Pública, XXIV(1), pp. 85-130.

Child, J. y D. Faulkner (1998), Strategies of Cooperation: Managing Alliances, Networks, and Joint Ventures, Oxford, Oxford University Press.

Hajer, M. y H. Wagennar (2003), Deliverative Policy Analysis: Understanding Governance in the Network Society, Cambridge University Press.

Innes, J. y D. Booher (2003), “Collaborative Policymaking: Governance Through Dialogue”, en M.A. Hajer y H. Wagenaar (eds.), Deliverative Policy Analysis: Understanding Governance in the Network Society, Cambridge, Cambridge University Press.

Klijn, E.H. (1998), "Policy Networks: An Overview”, en W.J.M. Kickert, E.H. Klijn y J.F.M. Koppenjan (eds.), Managing Complex Networks, Londres, Sage.

Lane, C. y R. Bachmann (1998), Trust Within and Between Organization, Oxford, Oxford University Press.

Mayntz, R. (1998), "New Challenges to Governance Theory”, Florence-Jean Monnet Chair Papers, 50.

Ostrom, E. (2015), Governing the Commons: The Evolution of Institutions for Collective Action, Cambridge, Cambridge University Press.

Pierre, J. Y G. Peters (2000), "Why the Concern with Governance Now?” en Governance, Politics and the State, Nueva York, St. Martin's Press.

Rhodes, R.A.W. (1997), Understanding Governance: Policy Networks, Governance, Reflexivity and Accountability, Nueva York, Open University Press.

Scott, J. (1999), Seeing Like the State: How Certain Schemes to Improve the Human Condition Have Failed, Nueva York, Yale University Press.

Stone, D. (2011), Policy Paradox: The Art of Political Decision Making, tercera edición, Nueva York, W.W. Norton \& Company.

Sumano, J.A. (2020), "Prevención del delito en la frontera norte a través de la administración pública deliberativa", Gestión y Política Pública, XXIX(1), pp. 67-96.

Tarragó, D. y Q. Brugué-Torruella (2015), “La administración deliberativa: De la eficacia y la eficiencia a la inteligencia, y de la burocracia a la innovación”, Textos para Discusión de la CEPAL, 58.

\section{GUÍA DE PREGUNTAS PARA LA DISCUSIÓN EN CLASE}

\section{Preguntas de análisis}

¿Cuáles fueron los principales retos que enfrentó María Elena Domínguez como subsecretaria de Prevención y Participación Ciudadana? 
¿Cuáles eran los principales actores involucrados en la prevención social de la violencia y la delincuencia en Nuevo León? ¿Qué intereses tenía cada uno?

¿Qué tipo de conflictos es posible observar a lo largo del caso?

¿Qué rol tienen los actores no gubernamentales en el desarrollo del caso?

¿Cuáles fueron las acciones que María Elena Domínguez decidió emprender para dar respuesta a dichos retos?

\section{Preguntas de acción}

¿Qué hubieras hecho diferente a María Elena Domínguez ante el escenario presentado?

¿Qué acciones sugieres para mejorar el impacto de los programas y acciones de prevención social de la violencia y la delincuencia?

¿Cómo se podría mejorar la coordinación entre los diferentes actores involucrados en la prevención social de la violencia y la delincuencia?

¿Cómo se podrían mejorar los mecanismos de deliberación respecto a la política de prevención social de la violencia y la delincuencia?

\section{Preguntas de reflexión}

¿De qué forma la gobernanza contribuye a resolver problemas complejos como la violencia y delincuencia?

¿Las respuestas a la violencia y delincuencia emanadas de la deliberación son más efectivas que las soluciones generadas en un esquema top-down?

¿Qué rol deben asumir los actores no gubernamentales en la coproducción de seguridad ciudadana?

¿Qué papel tienen la confianza y el diálogo en la solución de problemas complejos como la violencia y delincuencia?

José Andrés Sumano actualmente es catedrático Conacyt y profesor-investigador en el Colegio de la Frontera Norte. En el sector público ha sido titular del Centro Estatal de Prevención del Delito de Nuevo León, asesor del Comisionado Nacional de Seguridad, asesor del secretario de Seguridad Pública y director de Seguridad Internacional en el Cisen. Cuenta con un doctorado en Política Pública por el Instituto Tecnológico y de Estudios Superiores de Monterrey y una maestría en Administración Pública y Política Pública por la misma institución. 